\title{
ABRÉVIATIONS ET NOTATIONS
}

$:=$ : égal par définition.

cf. : confer, signifie « se reporter à ».

i.e. : id est, signifie « c'est-à-dire».

ln : notation normalisée pour le logarithme népérien.

$\mathbb{R}_{*}^{+}, \mathbb{R}_{+}^{*}$ ou $] 0,+\infty[$ : ensemble des réels strictement positifs.

$u^{+}$: partie positive du réel $u$.

$x=\left(x_{1}, \ldots, x_{n}\right)$ ou $x=\left(\xi_{1}, \ldots, \xi_{n}\right)$ : notation générique pour un vecteur de $\mathbb{R}^{n}$.

$u^{+}$signifie $\left(u_{1}^{+}, \ldots, u_{n}^{+}\right)$lorsque $u=\left(u_{1}, \ldots, u_{n}\right) \in \mathbb{R}^{n}$.

Lorque $u$ et $v$ sont deux vecteurs de $\mathbb{R}^{n}, u \leqslant v$ signifie $\ll u_{i} \leqslant v_{i}$ pour tout $i=1, \ldots, n \gg$.

$\left\{u_{k}\right\}$ ou $\left(u_{k}\right)$ : notations utilisées pour les suites indexées par des entiers naturels.

Pour une fonction $f$ différentiable en $x$ (resp. deux fois différentiable en $x$ ), $D f(x)$ désigne la différentielle (première) de $f$ en $x$ (resp. $D^{2} f(x)$ désigne la différentielle seconde de $f$ en $x$ ). Si la variable est réelle (et notée $t$ ), on utilise la notation $f^{\prime}(t)$ (resp. $f^{\prime \prime}(t)$ ) pour la dérivée de $f$ en $t$ (resp. la dérivée seconde de $f$ en $t$ ) [ce sont des éléments de l'espace d'arrivée et non des applications linéaires].

Pour une fonction numérique $f$ définie sur un ouvert $\mathcal{O}$ de $\mathbb{R}^{n}$, différentiable en $x \in \mathcal{O}$ (resp. deux fois différentiable en $x \in \mathcal{O}$ ), $\nabla f(x)$ (resp. $\nabla^{2} f(x)$ ) désigne le (vecteur) gradient de $f$ en $x$ (resp. la matrice hessienne de $f$ en $x$ ).

Lorsqu'elle existe, la dérivée directionnelle de $f$ en $x$ dans la direction $d$ est notée $f^{\prime}(x, d)$.

Pour une fonction vectorielle $f: \mathcal{O} \subset \mathbb{R}^{n} \rightarrow \mathbb{R}^{m}$ différentiable en $x \in \mathcal{O}, J f(x)$ désigne la matrice jacobienne de $f$ en $x$ (matrice à $m$ lignes et $n$ colonnes). 
$\mathcal{M}_{m, n}(\mathbb{R})$ : ensemble des matrices $(m, n)$ ( $m$ lignes et $n$ colonnes) à coefficients réels; $\mathcal{M}_{n}(\mathbb{R})$ est une abréviation de $\mathcal{M}_{n, n}(\mathbb{R})$.

$\left[a_{i j}\right]$ : matrice de terme général $a_{i j}$ (à la $i$-ème ligne et $j$-ème colonne).

diag $\left(\lambda_{1}, \ldots, \lambda_{n}\right)$ : matrice diagonale dont les éléments diagonaux sont $\lambda_{1}, \ldots, \lambda_{n}$.

$I_{n}$ (ou $I$ quand il n'y a pas d'ambiguïté) : matrice-unité de $\mathcal{M}_{n}(\mathbb{R})$, i.e. $\operatorname{diag}(1, \ldots, 1)$.

$A^{\top}$ ou ${ }^{t} A$ : transposée de $A \in \mathcal{M}_{m, n}(\mathbb{R})$ [les deux notations sont d'un usage très courant; par contre $A^{t}$ est à proscrire car génératrice de confusions].

Lorsque $A$ est inversible, $A^{-\top}$ désigne l'inverse de $A^{\top}$ (ou, ce qui revient au même, la transposée de $A^{-1}$ ).

$\operatorname{tr} A$ : trace de $A \in \mathcal{M}_{n}(\mathbb{R})$.

dét $A$ : déterminant de $A \in \mathcal{M}_{n}(\mathbb{R})$.

cof $A$ : matrice des cofacteurs de $A \in \mathcal{M}_{n}(\mathbb{R})$, i.e. celle dont le terme $(i, j)$ est $(-1)^{i+j}$ dét $A_{i j}$, où $A_{i j}$ est obtenue à partir de $A$ en enlevant la $i$-ème ligne et la $j$-ème colonne.

$\mathcal{S}_{n}(\mathbb{R})$ : ensemble des matrices de $\mathcal{M}_{n}(\mathbb{R})$ qui sont symétriques.

$\oplus$ symbolise la somme directe de sous-espaces vectoriels.

vect $\left\{v_{1}, \ldots, v_{k}\right\}$ : sous-espace vectoriel engendré par les vecteurs $v_{1}, \ldots, v_{k}$.

Sauf indication contraire, $\mathbb{R}^{n}$ est muni de sa base canonique; ainsi $\grave{a} A \in$ $\mathcal{M}_{m, n}(\mathbb{R})$ est canoniquement associée une application linéaire de $\mathbb{R}^{n}$ dans $\mathbb{R}^{m}$, d'où les notations $\operatorname{Ker} A, \operatorname{Im} A$, etc.

L'isomorphisme canonique de $\mathbb{R}^{n}$ sur $\mathcal{M}_{n, 1}(\mathbb{R})$ est celui qui à $x=\left(x_{1}, \ldots, x_{n}\right)$ associe la matrice unicolonne $X=\left[\begin{array}{c}x_{1} \\ \vdots \\ x_{n}\end{array}\right]$; des expressions comme $A X$ (ou $A x$ ) ne devraient pas arrêter l'étudiant-lecteur. Si, par exemple, $u$ et $v$ sont deux vecteurs de $\mathbb{R}^{n}$, uv ${ }^{\top}$ est une matrice carrée de taille $n$ dont le terme général est $u_{i} v_{j}$, alors que $u^{\top} v$ est la matrice-scalaire (ou scalaire) $\sum_{i=1}^{n} u_{i} v_{j}$.

$\langle\cdot, \cdot\rangle, \ll \cdot, \cdot \gg,(\cdot \mid \cdot)$ : notations utilisées pour les produits scalaires (dans des espaces euclidiens). Sauf indication contraire, $\langle\cdot, \cdot\rangle$ désigne dans $\mathbb{R}^{n}$ le produit scalaire usuel (celui qui à $x=\left(\xi_{1}, \ldots, \xi_{n}\right)$ et $y=\left(\eta_{1}, \ldots, \eta_{n}\right)$ associe $\langle x, y\rangle:=$ $\sum_{i=1}^{n} \xi_{i} \eta_{i}$, soit encore $x^{\top} y(c f$. supra $\left.)\right)$. Bien des problèmes d'optimisation se posent dans des espaces de matrices : si $X:=\mathcal{M}_{m, n}(\mathbb{R})$, le produit scalaire standard sur $X$ est défini par $\ll M, N \gg:=\operatorname{tr}\left(M^{\top} N\right)$. 
Soit $A \in \mathcal{M}_{n}(\mathbb{R}), u$ et $v$ des vecteurs de $\mathbb{R}^{n}: u^{\top} A v$ est un(e) (matrice-) scalaire égal(e) à (sa transposée) $u A^{\top} v$; un mécanisme plus commode d'utilisation et engendrant moins de fautes est d'écrire $\langle u, A v\rangle=\left\langle A^{\top} u, v\right\rangle$.

Si $l$ est une application linéaire d'un espace euclidien $(E, \ll \cdot, \cdot \gg)$ dans un autre espace euclidien $(F,\langle\cdot, \cdot\rangle)$, l'adjointe $l^{*}$ de $l$ est l'application linéaire de $F$ dans $E$ définie par

$$
\ll l^{*}(y), x \gg=\langle y, l(x)\rangle \text { pour tout }(x, y) \in E \times F .
$$

Si l'on représente l'ensemble des formes linéaires sur $E$ par $E$ via le produit scalaire $\ll \cdot, \cdot \gg$ (idem pour $F$ ), prendre l'adjointe de $l$ ou sa transposée revient au même. Lorsque $E$ et $F$ sont munies de bases orthonormales (comme c'est le cas des espaces euclidiens $\left(\mathbb{R}^{n},\langle\cdot, \cdot\rangle\right)$ munies de leurs bases canoniques), la matrice représentant l'adjointe de $l$ (= sa transposée) dans ces bases est la transposée de la matrice représentant $l$.

$\|\cdot\|$ : norme dans un espace vectoriel normé $X$. Si $X$ est muni d'un produit scalaire $\langle\cdot, \cdot\rangle$ (exemples typiques : $\mathbb{R}^{n}, \mathcal{M}_{m, n}(\mathbb{R})$ ), et en l'absence d'autres précisions, $\|\cdot\|$ désignera la norme dérivée du produit scalaire $($ i.e. $\|\cdot\|=\sqrt{\langle\cdot, \cdot\rangle}$ ). Lorsqu'interviennent à la fois des normes de vecteurs et de matrices, on évite les confusions en utilisant $\|\cdot\|$ pour les vecteurs et $|\|\cdot \mid\|$ pour les matrices).

int $S$ ou $\stackrel{\circ}{S}$ : intérieur de $S ; \bar{S}$ : adhérence de $S$;

fr $S$ : frontière de $S$.

$\bar{B}(x, r)$ : boule fermée de centre $x$ et de rayon $r$.

$\Delta_{n}$ : simplexe-unité de $\mathbb{R}^{n}$, c'est-à-dire l'ensemble des $\left(\alpha_{1}, \ldots, \alpha_{n}\right) \in \mathbb{R}^{n}$ tels que $\alpha_{1}+\ldots+\alpha_{n}=1$ et $\alpha_{i} \geqslant 0$ pour tout $i$ (coefficients qui servent dans la prise de combinaisons convexes).

$A \in \mathcal{S}_{n}(\mathbb{R})$ est dite (symétrique) semi-définie positive lorsque $\langle A x, x\rangle \geqslant 0$ pour tout $x \in \mathbb{R}^{n}$ [cette appellation est préférable à « positive » qui peut signifier, dans certains cas, «à coefficients positifs »]. On désignera par $\mathcal{P}_{n}(\mathbb{R})$ l'ensemble des matrices semi-définies positives de taille $n$.

$A \in \mathcal{S}_{n}(\mathbb{R})$ est dite (symétrique) définie positive lorsque $\langle A x, x\rangle>0$ pour tout $x \neq 0$ de $\mathbb{R}^{n}$ (ce qui revient à : $A$ semi-définie positive et inversible). On désignera par $\dot{\circ}_{n}(\mathbb{R})$ l'ensemble des matrices définies positives de taille $n$.

Le caractère de semi-définie ou définie positivité ne sera considéré dans ce recueil que pour des matrices symétriques. 
This page intentionally left blank 